\title{
Proteasome Inhibition Diminishes the Formation of Neutrophil Extracellular Traps and Prevents the Death of Cardiomyocytes in Coculture with Activated Neutrophils during Anoxia-Reoxygenation
}

\author{
Denis O. Pashevin Vasyl S. Nagibin Lesya V. Tumanovska Alex A. Moibenko \\ Victor E. Dosenko
}

Department of General and Molecular Pathophysiology, Bogomoletz Institute of Physiology,

National Academy of Science of Ukraine, Kiev, Ukraine

\section{Key Words \\ Neutrophil extracellular traps · Proteasome . \\ Cardiomyocytes $\cdot$ Anoxia-reoxygenation}

\begin{abstract}
Objective: Polymorphic mononuclear neutrophils (PMN) are very important cells participating in nonspecific defense of the organism. Among their well-known functions, the formation of neutrophil extracellular traps (NET) is interesting and potentially dangerous for the mechanisms of other cells. Ubiquitin-dependent proteasomal proteolysis is a very important regulator of all cellular activities, but the role of proteasomal proteolysis in NET formation has not been investigated. Methods: We performed experiments with PMN activated to form NET with phorbol 12-myristate 13-acetate (PMA) and the application of a proteasome inhibitor. We also added activated neutrophils to primary culture of isolated rat neonatal cardiomyocytes with or without anoxia-reoxygenation modeling. Results: The data obtained show that proteasomes participate in NET formation and proteasome inhibitors facilitate the blocking of the NET program. The percentage of NET after PMA application was 70.8 \pm 7.2 and the proteasome inhibitor decreased this amount to $4.7 \pm$ $0.9 \%$. In coculture with cardiomyocytes during anoxia-reox-
\end{abstract}

ygenation, this effect prevented cardiac cell death induced by activated PMN. The stimulation of NET formation by PMA in coculture with isolated cardiomyocytes led to an increase in the number of necrotic cardiomyocytes of up to $33.1 \pm$ $12.9 \%$ and a corresponding decrease in living cardiomyocytes to $66.9 \pm 12.9 \%$. The number of living cardiomyocytes in coculture after incubation with both PMA and proteasome inhibitor was $76.6 \pm 13.3 \%(p<0.05)$, and the number of necrotic cardiomyocytes was $23.4 \pm 13.3 \%(p<0.05)$. Conclusion: Proteasome inhibition blocks NET formation and prevents cardiomyocyte necrosis in coculture with activated neutrophils.

(c) 2015 S. Karger AG, Basel

\section{Introduction}

The nonspecific defense of the organism is a monumental system that includes many different factors and processes, and it features in almost all pathologies. One of the very important cell populations participating in this mechanism are polymorphic mononuclear neutrophils (PMN). These cells can perform phagocytosis, exocytosis of enzymes, production of free radicals, etc., and are a main component of secondary alteration in inflamma-

\section{KARGER 125}

(c) 2015 S. Karger AG, Base

$1015-2008 / 15 / 0826-0290 \$ 39.50 / 0$

E-Mail karger@karger.com

www.karger.com/pat
Vasyl S. Nagibin, $\mathrm{MD}, \mathrm{PhD}$

Department of General and Molecular Pathophysiology

Bogomoletz Institute of Physiology, National Academy of Science of Ukraine

Bogomoletz Street 4, Office 326, Kiev 01024 (Ukraine)

E-Mail nagibin@biph.kiev.ua 
tion of different genesis including ischemia-reperfusion injury [1]. In 2004, Brinkmann et al. [2] described a new type of PMN action, i.e. the formation of so-called neutrophil extracellular traps (NET). This phenomenon is mostly considered as a specific type of programmed cell death called NETosis [3]. During this process, the DNA of PMN appears in the extracellular space without fragmentation and forms nets that can trap bacteria or other pathogens. NET also contain histones (mainly histone H1) and active enzymes such as myeloperoxidase, elastase, cathepsin G, gelatinase, etc. [4]. This phenomenon is not limited only to the infection process. Studies in recent years show the great importance of NET in the pathogenesis of tumors [5], autoimmune disorders [6], thrombus formation [7], etc. De Boer et al. [7] observed NET in thrombi from coronary vessels of patients suffering from myocardial infarction by the colocalization of histone $\mathrm{H} 1$, myeloperoxidase and neutrophil elastase. On the other hand, it is well known that PMN infiltrate myocardial tissue during ischemia-reperfusion and can cause secondary alteration and the spreading of necrosis. Barrett et al. [8] established that perfusion of isolated hearts with a solution containing PMN significantly increases ischemic-reperfusion damage and the blockade of cell adhesion molecules abolishes this effect. There is still no information about the direct role of NET formation in myocardial infarction. We hypothesize that NETosis has a big effect on this pathological process via damage to cardiomyocytes by myeloperoxidase, elastase and free-radical formation.

Ubiquitin-dependent proteasomal proteolysis is a very important regulator of all cellular activities. Some proteins that maintain the DNA in chromosomes and regulate its status, especially the histones ( $\mathrm{H} 2 \mathrm{a}$ and $\mathrm{H} 2 \mathrm{~b})$, are always present in the nucleus in a monoubiquitinated state [9]. According to Knepper-Nicolai et al. [10], the proteasome is crucial for the constitutive apoptosis of PMN. It is also known from previous investigations that proteasome inhibitors effectively prevent ischemic reperfusion injury and damage to the heart caused by PMN [11]. The antiphlogistic effect of proteasome inhibitors also appears in many publications [12,13]. It is achieved particularly by a decrease of degradation of the nuclear factor kappa B (NF-kB) inhibitor. Argentine researchers have established that some structural inhibitors of NF- $\mathrm{KB}$ prevent NETosis [14]. However, the role of proteasomal proteolysis in NET formation has not been investigated.

Our previous data show the protective effect of proteasome inhibitors on cardiomyocytes at the modeling of anoxia-reoxygenation. Against a background of proteasome inhibitors, the application of anoxia-reoxygenation led to fewer amounts of necrotic cells in a primary culture of rat neonatal cardiomyocytes [15]. However, there is still a lack of data about the proteasome-dependent prevention of cardiomyocyte death due to secondary alteration.

Based on this, we checked the effect of the proteasome inhibitor on NET formation and established that clastolactacystin $\beta$-lactone (CL), that can specifically inhibit all 3 proteolytic activities of the proteasome [16], diminishes the formation of NET induced by phorbol 12-myristate 13-acetate (PMA). At the modeling of anoxia-reoxygenation, it was shown that proteasome inhibition prevents NET-induced cardiomyocyte death. The data that we obtained support our hypothesis about the participatioin of proteasomes in the formation of NET and the role of these processes in cardiomyocyte damage.

\section{Materials and Methods}

\section{Solutions and Drugs}

RPMI medium, FBS, antibiotics, Hoechst 33342, propidium iodide (PI), PMA, CL, DNAse Percoll, Hanks balanced salt solution (HBSS), glucose, salts and antibodies were purchased from Sigma Chemical Co., St Louis, Mo., USA. Free DNA determination was performed using the Quant-iT PicoGreen dsDNA assay kit (Invitrogen).

\section{PMN Isolation}

To obtain rat PMN, the blood from adult (3-month-old) Wistar rats was collected and stabilized by EDTA in monovettes (Sarstedt), mixed with $0.9 \% \mathrm{NaCl}$ in a ratio of $1: 1$ and carefully laminated on the Percoll gradient with layer densities of 45,54 and $63 \%$.

After a gradient centrifugation of the blood $(3,000 \mathrm{rpm}$ for 15 min) PMN were detected between the second and third layers. The cells were collected and additionally purified by erythrocyte lysis with ACK buffer. The granulocytes were then washed with HBSS buffer and diluted in RPMI media with $10 \%(\mathrm{v} / \mathrm{v})$ heat-inactivated FBS. The PMN were plated in the 24 -well dish or in the cell culture Petri dish with a density of 100,000 cells $/ \mathrm{cm}^{2}$.

\section{Stimulation and Detection of NET}

To stimulate NET formation, $20 \mathrm{nM}$ of PMA were added to cell cultures for $3 \mathrm{~h}$. To check if proteasomes participate in NET formation, the proteasome inhibitor CL was added at a concentration of $100 \mathrm{nM}$. Thus, 4 groups of cells were in used in the experiment: control neutrophils, PMN stimulated by PMA, PMN with the proteasome inhibitor and PMN under the influence of both PMA and the proteasome inhibitor. The same protocol was used for the coculture of rat neonatal cardiomyocytes and PMN including the step of anoxia-reoxygenation (see below).

Detection of NET was performed using a fluorescence microscope and a fluorogenic dye, Hoechst 33342 (biz-benzamide), that directly stains chromatin $(3.75 \mathrm{mM})$. Additionally, we measured the concentration of DNA released in the culture media; for this, DNAse 1 ( 5 units/ml) was added to the culture of PMN. After 10 
min of incubation, the DNAse reaction was stopped by adding EDTA ( $5 \mathrm{mM})$. The concentration of DNA was measured on a Hitachi 4000 spectrophotometer with a Quant-iT PicoGreen dsDNA assay kit (Invitrogen) according to the manufacturer's manual.

NET formation was also confirmed by detecting the extracellular colocalization of DNA and myeloperoxidase, the enzyme specific for PMN. It was done by fluorescence microscopy using Hoechst 33342 together with a polyclonal antibody specific for rat myeloperoxidase delivered in rabbits and Alexa Fluor 647-conjugated goat anti-rabbit secondary antibodies.

\section{Cardiomyocyte Isolation, Cultivation and Cocultivation} with PMN

To prepare the coculture of PMN with cardiomyocytes, the rat neonatal cardiomyocytes were isolated from the ventricular myocardium of 2-day-old Wistar rats by enzymatic digestion according to Reinecke et al. [17] with modifications. The amount of living and necrotic cells was determined by staining with $0.2 \%$ Trypan blue solution and was $90-95 \%$ and 5-10\%, respectively. To avoid fibroblast contamination, $2 \mathrm{~h}$ of preplating on cell culture dishes was performed. The cardiomyocytes obtained were plated at a density of $120,000 \mathrm{~cm}^{2}$. Cells were cultivated for $24 \mathrm{~h}$ in DMEM/ medium 199 (4:1), supplemented with $10 \%$ calf serum, $4.2 \mathrm{~mm}$ $\mathrm{Na}_{2} \mathrm{CO}_{3}, 15 \mathrm{mM}$ HEPES and antibiotics (streptomycin $100 \mathrm{~g} / \mathrm{ml}$, gentamycin $0.05 \mathrm{mg} / \mathrm{ml}$ and penicillin $100 \mathrm{U} / \mathrm{ml}$ ) at $37^{\circ} \mathrm{C}$ in a $5 \%$ $\mathrm{CO}_{2}$ atmosphere. After $24 \mathrm{~h}$, fresh isolated PMN or PMN activated by PMA were added to the rat neonatal cardiomyocyte culture in the ratio $1-1.2: 1-0.8$.

\section{Measurement of Proteasome Activity}

Cells were collected from the Petri dish, sonicated and centrifugated at 5,000 rpm for $10 \mathrm{~min}$. Supernatant was incubated in a buffer consisting of $25 \mathrm{~mm}$ Tris- $\mathrm{HCl}(\mathrm{pH} \mathrm{7.5)}$ and $1 \mathrm{mM}$ dithiothreitol. Fluoropeptide substrate Suc-Leu-Leu-Val-Tyr-7-amido-4-methylcoumarin was used to measure the chymotrypsin-like activity of the proteasome; Boc-Leu-Ser-Thr-Arg-7-amido-4methylcoumarin was used for the trypsin-like activity and CBZLeu-Leu-Glu-7-amido-4-methylcoumarin for the peptidyl-glutamyl peptide-hydrolyzing activity. After incubation for $30 \mathrm{~min}$ (for trypsin-like activity) or $1 \mathrm{~h}$ (for other activities) with 1 of these fluorogenic peptides (at concentration $6 \mu \mathrm{M}$ ), the fluorescence of the reaction products was monitored at $380 \mathrm{~nm}$ excitation and 440 $\mathrm{nm}$ emission using free 7-amino-4-methylcoumarin (AMC) as a standard on a Hitachi 4000 spectrofluorometer. The reaction was performed in the absence and presence of selective proteasome inhibitors, i.e. CL $(2.5 \mu \mathrm{M})$ or MG132 $(5 \mu \mathrm{M})$, to differentiate between nonproteasome and proteasome-mediated peptide hydrolysis. The percentage of inhibition of the respective substrate hydrolysis after the use of each inhibitor was evaluated as the proteasome activity and expressed as $\mathrm{nM}$ of $\mathrm{AMC} / 10^{6}$ cells/min.

\section{Determining Cardiomyocyte Death and Anoxia-Reoxygenation} Modeling

In the coculture of rat neonatal cardiomyocytes and PMN, we determined not only NET formation but also the necrotic death of cardiomyocytes. We used PI, a chromatin-specific dye that cannot pass through the cell membrane. The double staining with PI and Hoechst 33342 allowed us to differentiate cardiomyocytes from PMN by the shape of the nuclei and also the living cardiomyocytes (Hoechst 33342 positive) from the necrotic ones (PI positive).
For anoxia-reoxygenation, the coculture of rat neonatal cardiomyocytes and PMN underwent 30 min of incubation in an airtight jar from which the $\mathrm{O}_{2}$ was flushed with a gas mixture containing $5 \% \mathrm{CO}_{2}$ and $95 \% \mathrm{Ar}$, followed by replacement of the media and $3 \mathrm{~h}$ of incubation in a $5 \% \mathrm{CO}_{2}$ incubator.

\section{Statistics}

Statistical analysis was performed with Origin 7.0 software. All data were expressed as means $( \pm S D)$ of replicate experiments performed in each assay. Statistical differences were evaluated using the Student t test. $\mathrm{p}<0.05$ was taken to indicate a statistically significant difference.

\section{Bioethics}

This investigation conformed to the local ethical committee guidelines for animal research. All animal experiments were in compliance with the statements of the European Union regarding the handling of experimental animals.

\section{Results}

\section{Determination of Proteasome Activities in \\ Isolated PMN}

All 3 proteolytic activities of the proteasome were determined in the isolated PMN: trypsin-like activity was $0.21 \pm 0.6$, chymotrypsin-like $-4.4 \pm 0.4$ and peptidyl-glutamyl peptide-hydrolyzing activity $-0.21 \pm 0.4 \mathrm{nmol}$ AMC $/ 10^{6}$ cells/min. We confirmed that CL application significantly inhibited all 3 proteolytic activities of the proteasome in PMN: trypsin-like activity was reduced 1.9-fold, chymotrypsin-like activity 2.2-fold and peptidyl-glutamyl peptide-hydrolyzing activity 2.6-fold ( $\mathrm{p}<$ 0.05 for all activities). These data show that in isolated PMN all 3 peptidase activities of proteasome are detected at a high level and CL effectively inhibits these activities.

\section{Formation of NET by PMN under the Influence of \\ Proteasomal Inhibitor: Fluorescence Microscopy}

In the control group, we observed intact PMN cells under fluorescence microscopy (fig. 1a). After $3 \mathrm{~h}$ of incubation with PMA, neutrophils were activated and formed NET (fig. 1b, c). Cells with highly condensed chromatin without nuclei segregation and fragmentation were detected. Some had no condensed chromatin and the nuclei in these were bigger than in other cells and were round in shape with no signs of segmentation or fragmentation (fig. 1d). It is should be mentioned that myeloperoxidase was not detected in combination with chromatin in these cells (fig. 2c, d) and these nuclei were not stained with PI, meaning that the integrity of the PMN membranes remained intact. It may be that neutrophils did not complete NET formation. The population of neu- 

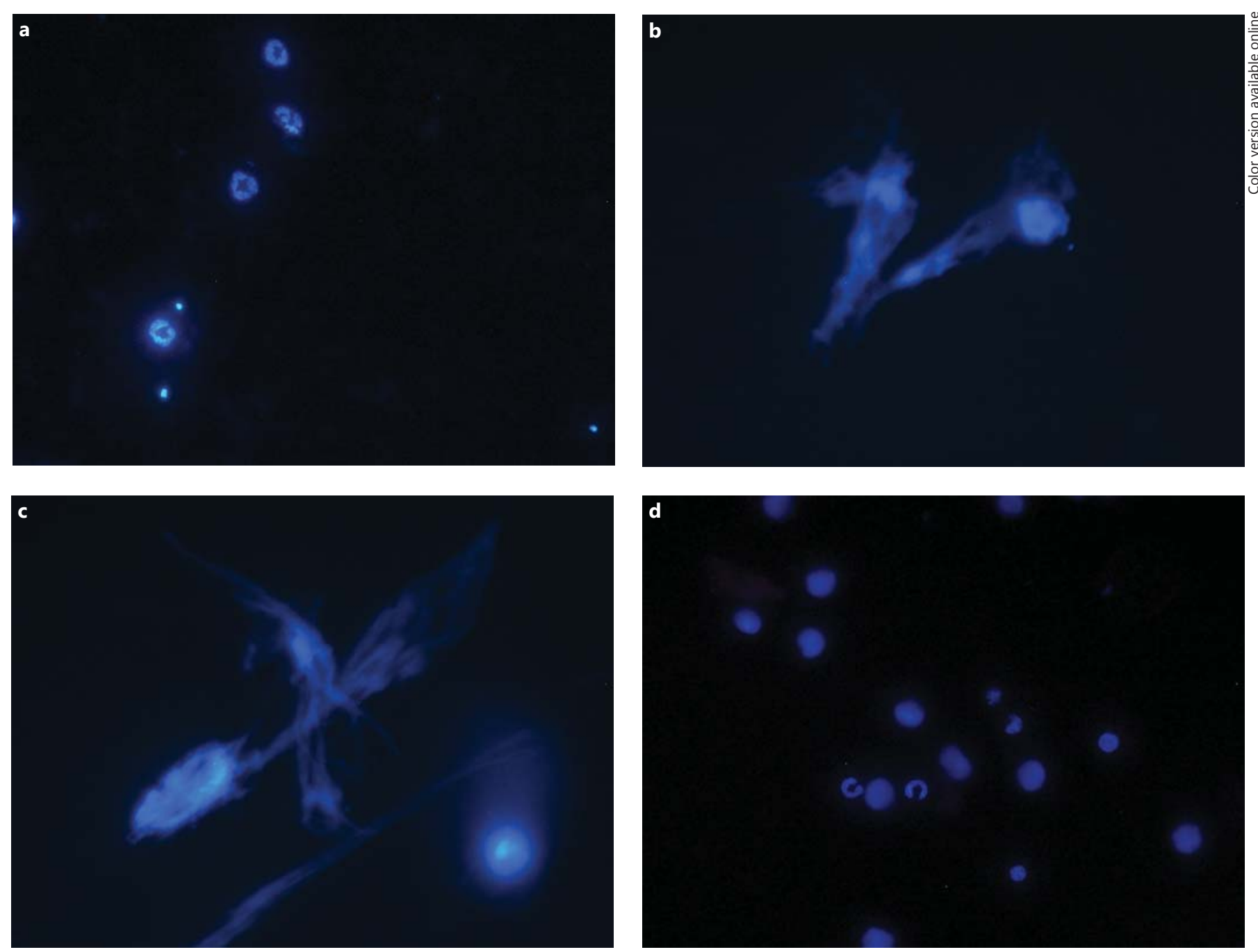

Fig. 1. Fluorescence microscopy of isolated PMN stained with Hoechst 33342 . a Control cells. $\times 40$. b, c NET after $3 \mathrm{~h}$ of incubation with PMA. $\times 100$. d Impairment of NET formation after $3 \mathrm{~h}$ of incubation with both proteasome inhibitor and PMA. $\times 40$.

trophils was large after the incubation of PMN with the proteasome inhibitor and they were observed in almost all fields (fig. 1d). Thus, PMN with unfinished, impaired NET formation revealed that the proteasome inhibitor blocks the program of NET formation. Necrotic PMN were not detected in any experimental group.

To confirm that the release of DNA was due to NET formation, we performed immunochemistry of the neutrophils to indicate the colocolization of neutrophil myeloperoxidase and DNA chains. This intracellular enzyme marked for neutrophils was observed as a component of NET after PMA stimulation (fig. 2b). As mentioned above, myeloperoxidase was not detected in combination with

Proteasome Inhibitor Blocks NETosis and Prevents Cardiomyocyte Death chromatin after proteasome inhibitor application when a lot of PMN did not have segregated nuclei (fig. 2c, d).

The amount of living PMN in the control was $97.2 \pm$ $2.5 \%$ and $2.8 \pm 2.5 \%$ of control cells had formed NET. After PMA stimulation, the percentage of NET was $70.8 \pm$ $7.2(\mathrm{p}<0.01)$ and the living cells amounted to $27.2 \pm 7.2 \%$ $(\mathrm{p}<0.01)$. The rest of the cells after PMA stimulation $(1.7 \pm 0.3 \%)$ had lost the characteristic segmentation of nuclei and were marked by diffused chromatin distribution, a sign of impaired NET formation, in the opinion of some authors. CL also induced NET formation in the culture of PMN cells, but the amount of NET was 15 times less than in the PMA group $(\mathrm{p}<0.01)$. At the same time, 

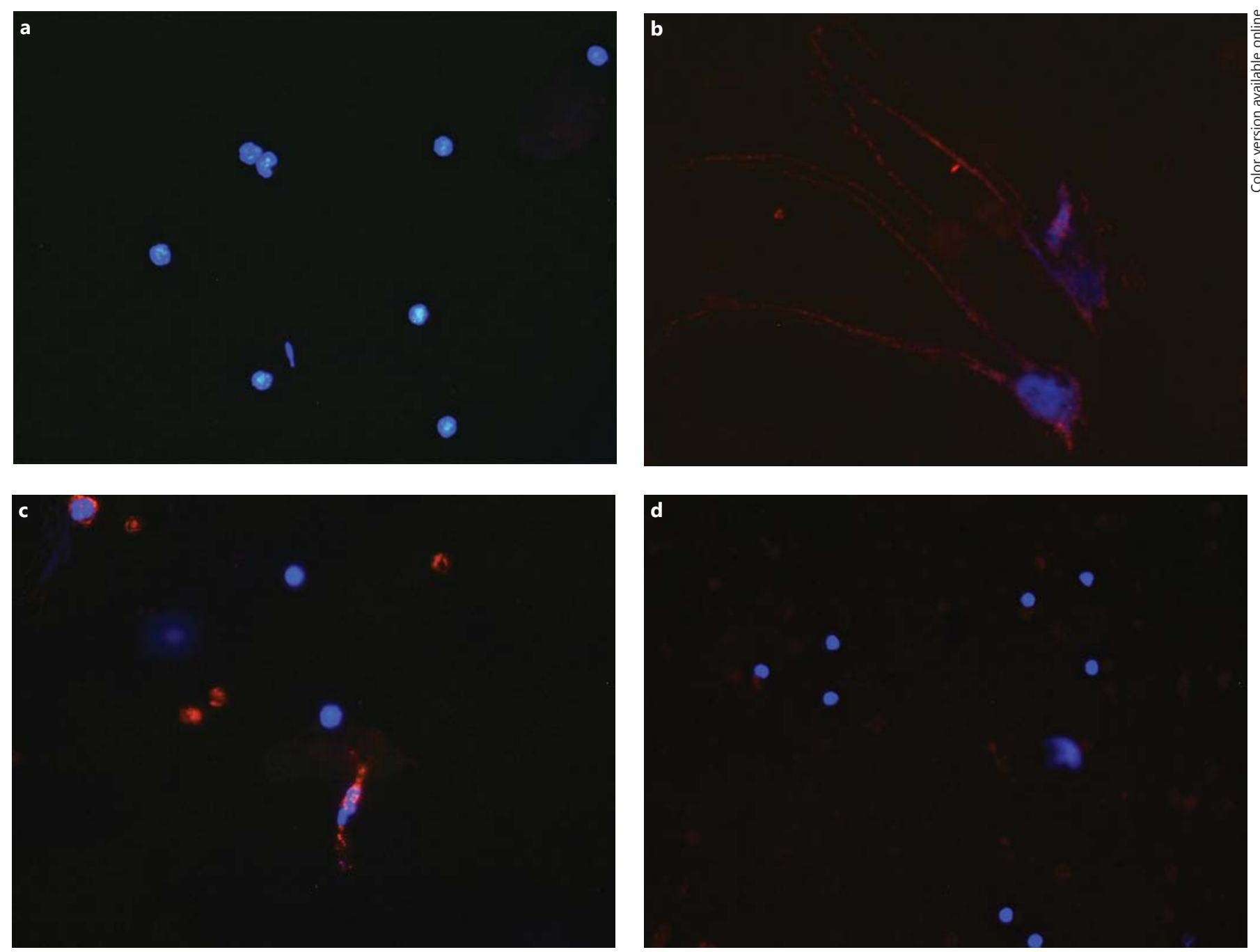

Fig. 2. Fluorescence microscopy of isolated PMN stained with Hoechst 33342 (blue) and antibodies against myeloperoxidase (red). a Control. b NET after 3 h of incubation with PMA. c Cells after $3 \mathrm{~h}$ of incubation only with proteasome inhibitor. $\mathbf{d}$ Cells after $3 \mathrm{~h}$ of incubation with both PMA and proteasome inhibitor. $\times 40$. Colors refer to the online version only.

the inhibition of the proteasome provoked the increase of PMN with impairment of NET formation. The percentage of living cells and NET in isolated PMN culture after proteasome inhibitor application was thus $38.8 \pm 11.1$ and $4.7 \pm 0.9$, respectively. The rest, $56.5 \%$, constituted neutrophils with impairment of NET formation. However, the most interesting results were obtained after application of proteasome inhibitor together with PMA. The number of NET in this experimental group was 1.8 times less than with PMA stimulation only $(\mathrm{p}<0.01)$ and totaled $38.7 \pm 4.9 \%$. The percentage of living cells was $25.4 \pm 4.9$, and the amount of neutrophils with impaired NET formation was $35.9 \pm 4.9 \%$ (fig. 3 ).

\section{The Concentration of DNA Released from PMN} due to NET

The concentration of DNA released into the culture media from PMN during NET formation was $2.8 \pm 0.34$ $\mathrm{ng} / \mathrm{ml}$ in the control, $8.4 \pm 1.2 \mathrm{ng} / \mathrm{ml}$ after PMA stimulation ( 3 times more; $\mathrm{p}<0.05$ vs. the control) and $4.3 \pm 1.1$ $\mathrm{ng} / \mathrm{ml}$ after incubation with both PMA and proteasome inhibitor (1.95 times less vs. the PMA-stimulated group; $\mathrm{p}<0.05$; fig. 4).

All these data directly show that proteasome inhibition prevents NET formation leading to an increased amount of PMN with impaired NET formation. 


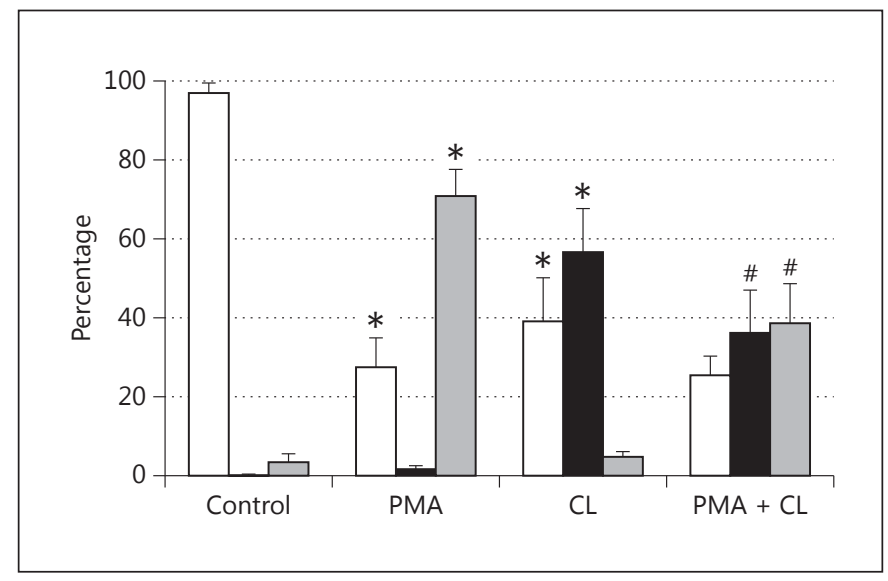

Fig. 3. Percentages of living neutrophils (white columns), PMN with impairment of NET formation (black columns) and NET (shaded columns) in a culture of PMN under the influence of a NET activator, PMA and proteasome inhibitor. ${ }^{*} \mathrm{p}<0.01 \mathrm{com}-$ pared to control; ${ }^{\#} \mathrm{p}<0.01$ compared to PMA group.

Cocultivation of Cardiomyocytes with Activated PMN

Another part of our work was the investigation of the influence of NET formation on cardiomyocyte survival in coculture under anoxia-reoxygenation conditions. The anoxia-reoxygenation of the cardiomyocytes with PMA but without PMN led to an increase of necrotic cells amounting to $38.5 \pm 16 \%$.

However, changes in the percentage of living and necrotic cardiomyocytes were more prominent in the coculture with PMN. In the control coculture, $81.1 \pm 10 \%$ of the cardiomyocytes were living and $18.9 \pm 10 \%$ were necrotic. The stimulation of NET formation by PMA led to an increase in necrotic cardiomyocytes of up to $33.1 \pm$ $12.9 \%(\mathrm{p}<0.05)$ and a corresponding decrease in living cardiomyocytes amounting to $66.9 \pm 12.9 \%(\mathrm{p}<0.05)$. The CL application had no influence on the percentage of living and necrotic cardiomyocytes in the coculture with PMN (79.3 \pm 13 and $20.7 \pm 13 \%$, respectively) but prevented necrotic cardiomyocytes death after stimulation of neutrophils by PMA. Living cardiomyocytes in coculture after incubation with both PMA and proteasome inhibitor amounted to $76.6 \pm 13.3 \%(\mathrm{p}<0.05)$ and necrotic cardiomyocytes amounted to $-23.4 \pm 13.3 \%(\mathrm{p}<0.05)$ when compared to the PMA coculture group.

At modeling of anoxia-reoxygenation, the percentage of living and necrotic cardiomyocytes in coculture was $72.5 \pm 7.3$ and $27.5 \pm 7.3 \%$, respectively $(\mathrm{p}<0.05)$. The stimulation of PMN with PMA under anoxia-reoxygenation conditions led to a decrease in living cardiomyo-

Proteasome Inhibitor Blocks NETosis and Prevents Cardiomyocyte Death

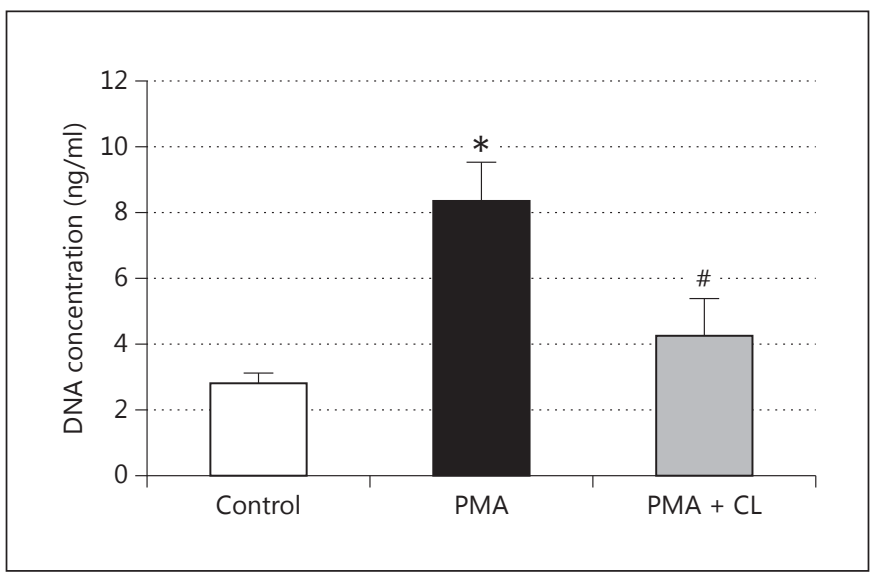

Fig. 4. The concentration of DNA released from PMN in culture media. ${ }^{*} \mathrm{p}<0.01$ compared to control; ${ }^{\#} \mathrm{p}<0.05$ compared to PMA group.

cytes $(52.5 \pm 5 \%)$ and an increase in necrotic ones (47.5 \pm $5 \%$; $<0.05$ vs. anoxia-reoxygenation group). The application of CL on coculture at modeling of anoxia-reoxygenation had no significant influence on the amount of living and necrotic cardiomyocytes. The combination of proteasome inhibitor with PMA at anoxia-reoxygenation modeling led to an increase of living cardiomyocytes up to $62.3 \pm 4.5 \%$ and a corresponding decrease in the population of necrotic cells to $37.7 \pm 4.5 \%$ in comparison with the anoxia-reoxygenation and PMA group $(\mathrm{p}<0.05$; table 1). Apoptotic cardiomyocytes were not detected in any experimental group.

These data illustrate that PMA itself can provoke cardiomyocyte death but that, in coculture with PMN, the amount of necrotic cardiomyocytes is much higher because of PMN activation. The inhibition of the proteasome prevents cell death after anoxia-reoxygenation not only by a direct influence on cardiomyocytes but also by preventing NET formation by PMN.

\section{Discussion}

Our experiments indicate that the proteasome takes part in NET formation. Some previous investigations support this. It is known from the work of Yost et al. [18] that embryonic PMN impair NET formation. In 2014, based on quantitative proteomics, Zhu et al. [19] proved that 7 subunits of proteasome were downregulated in 
Table 1. The percentage of living and necrotic cardiomyocytes in coculture with PMN under the influence of PMA, proteasomal inhibitor and anoxia-reoxygenation modeling

\begin{tabular}{|c|c|c|}
\hline & Living & Necrotic \\
\hline Control coculture & $81.06 \pm 10 \%$ & $18.9 \pm 10 \%$ \\
\hline $\mathrm{n}$ & 15 & 15 \\
\hline Control coculture $+\mathrm{AR}$ & $72.5 \pm 7.3 \%$ & $27.5 \pm 7.3 \%$ \\
\hline $\mathrm{n}$ & 13 & 13 \\
\hline $\mathrm{p}$ & 0.02 & 0.02 \\
\hline PMA coculture & $66.9 \pm 12.9 \%$ & $33.1 \pm 12.9 \%$ \\
\hline $\mathrm{n}$ & 15 & 15 \\
\hline $\mathrm{p}$ & 0.002 & 0.002 \\
\hline PMA coculture + AR & $52.5 \pm 5 \%$ & $47.5 \pm 5 \%$ \\
\hline $\mathrm{n}$ & 11 & 11 \\
\hline $\mathrm{p}^{1}$ & 0.0001 & 0.0001 \\
\hline $\mathrm{p}^{2}$ & 0.002 & 0.002 \\
\hline Control coculture $+\mathrm{CL}$ & $79.25 \pm 13 \%$ & $20.75 \pm 13 \%$ \\
\hline $\mathrm{n}$ & 8 & 8 \\
\hline $\mathrm{p}$ & 0.7 & 0.7 \\
\hline Control coculture $+\mathrm{AR}+\mathrm{CL}$ & $65.8 \pm 4.4 \%$ & $34.2 \pm 4.4 \%$ \\
\hline $\mathrm{n}$ & 10 & 10 \\
\hline $\mathrm{p}^{1}$ & 0.02 & 0.01 \\
\hline $\mathrm{p}^{3}$ & 0.007 & 0.007 \\
\hline PMA coculture + CL & $76.6 \pm 13.3 \%$ & $23.4 \pm 13.3 \%$ \\
\hline $\mathrm{n}$ & 15 & 15 \\
\hline $\mathrm{p}$ & 0.3 & 0.3 \\
\hline $\mathrm{p}^{2}$ & 0.05 & 0.05 \\
\hline PMA coculture + AR + CL & $62.3 \pm 4.5 \%$ & $37.7 \pm 4.5 \%$ \\
\hline $\mathrm{n}$ & 11 & 11 \\
\hline $\mathrm{p}^{1}$ & 0.0006 & 0.0006 \\
\hline $\mathrm{p}^{4}$ & 0.001 & 0.0001 \\
\hline
\end{tabular}

$\mathrm{AR}=$ Anoxia-reoxygenation modeling. $\mathrm{p}=$ Compared to control; $\mathrm{p}^{1}=$ compared to control + AR group $; \mathrm{p}^{2}=$ compared to PMA group; $\mathrm{p}^{3}=$ compared to control $+\mathrm{CL}$ group; $\mathrm{p}^{4}=$ compared to $\mathrm{PMA}+\mathrm{AR}$ group.

neonatal PMN when compared to adults. In our experiments, we obtained direct data about the effect of proteasome inhibitors on the realization of NET: a decrease in NET and impaired NET formation show blocking of this process at the stage of DNA extrusion in the extracellular space.

Yost et al. [18] obtained similar data for cord PMN: 'Organized NETs were not detected by live cell imaging ... In some studies there were bright, focal "hot spots" of staining by the cell-impermeable DNA dye suggesting local pericellular DNA extrusion, which is thought to be an initial step in complex extracellular NET formation'.

In our work, we observed PMN with highly condensed chromatin without nucleus segregation and fragmenta- tion. Some did not have condensed chromatin and the nuclei were bigger than in other cells, round in shape and with no signs of segmentation or fragmentation. So we could interpret them to be PMN with impaired NET formation and, because of most of them appeared after proteasome inhibitor application, the role of the proteasome in the realization of NET mechanisms could be supposed.

On the other hand, it is known that the proteasome has great importance in apoptosis development and that proteasome inhibition provokes apoptotic cell death in many cell types. It has been shown that several genes involved in ubiqutin-proteasome proteolysis are downregulated during phagocytosis-induced apoptosis of PMN [20]. The proteasome is also necessary for the realization of the constitutive apoptosis of PMN during their maturity and aging [10]. The role of the proteasome in the apoptosis of $\mathrm{PMN}$ of different genesis is usually explained by the proteasome-dependent activation of NF- $\kappa \mathrm{B}$ [21]. Apoptosis of PMN has morphological peculiarities under fluorescence microscopy that are close to those that we have described above, but fragmentation of DNA as a key marker of the final step in PMN apoptosis $[20,21]$ was not observed in our experiments.

The interaction between cardiomyocytes and PMN is mostly discussed in terms of cytokines and chemokines, endothelial permeability in heart microvessels and a socalled proinflammatory cardiomyocyte phenotype [22, 23]. This phenotype is characterized by different proinflammatory cytokine synthesis and secretion [24]. On the other hand, Barrett et al. [8] established that perfusion of an isolated heart with a solution containing PMN significantly increases ischemic-reperfusion damage and that the blockade of cell adhesion molecules abolishes this effect. It means that PMN can shift cardiomyocytes to a proinflammatory phenotype and then after this pass through the endothelium using chemokinesis. PMN can injure cardiomyocytes by enzyme exocytosis, phagocytosis, etc. [25]. In our work, we showed that direct damage of cardiomyocytes as a result of NET formation is also possible.

It is also interesting that we did not observe apoptosis of cardiomyocytes in the coculture. It was shown, in the recently published work of Carmona-Rivera et al. [26], that NET formed during systemic lupus erythematosus induce apoptosis of human umbilical vein endothelium in culture. We think that NET formation has different effects on different cell types under different conditions; thus, in the culture of rat neonatal cardiomyocytes under hypoxia-reoxygenation, it provokes necrosis in contrast to human umbilical vein endothelium in systemic lupus 
erythematosus. It is also can be explained by different number of activated neutrophils involved in coculture experiments. Higher amount of neutrophils can provoke necrosis due to the large externalized concentration of proteolytic enzymes.

There are not a lot of data on the role of NET in ischemia. In the work of Ge et al. [27], in situ NET formation was analyzed during surgical modeling of ischemia-reperfusion injury of the left ventricle, and the anatomic 'no re-flow' area and infarct size were evaluated immediately after $3 \mathrm{~h}$ of reperfusion. It was shown that, compared with the ischemia-reperfusion controls, the group of Wistar rats treated with DNAse and recombinant tissue-type plasminogen activator exhibited reduced NET density and a 'no-flow' area in the ischemic region as well as a reduced infarct size. Savchenko et al. [28] demonstrated that extracellular chromatin released through NETosis exacerbates myocardial ischemic-reperfusion injury. Oklu et al. [29] demonstrated that hind-limb ischemiareperfusion injury is reduced in Toll-like receptor-4 mutant mice that are characterized by decreased NET formation.

Our previous results showed that in a culture of isolated cardiomyocytes without PMN after anoxia-reoxy- genation modeling, the number of necrotic cells increased almost 2-fold [15]. In addition, inhibition of the proteasome did not lead to a significant increase of necrotic cardiomyocytes compared to control, but the application of a proteasome inhibitor at anoxia-reoxygenation decreased the number of necrotic cells compared to in the anoxia-reoxygenation group. The cardioprotective effects of proteasome inhibition during myocardial infarction are also known; these are summarized in the review of $\mathrm{Yu}$ and Kem [30]. All these data indicate that proteasome inhibitors prevent damage of cardiomyocytes during ischemia-reperfusion, but not only by their direct influence on cardiomyocyte metabolism. In the whole organism, this effect is mediated by the prevention of PMN activation and its consequences such as thrombi formation and other mechanisms; the direct injury of cardiomyocytes due to NET formation has a very important place amongst these. Our experiments confirmed this hypothesis: the incubation of activated PMN with proteasome inhibitors reduces cardiomyocyte death during cocultivation under anoxia-reoxygenation conditions. This provides possibilities for further study of NETosis as well as for the therapeutic prevention of secondary alterations during myocardial infarction.

\section{References}

1 Dun Y, Zhi J, Sun H, Zhao R, Zhao Z: Activated polymorphonuclear leukocytes induce cardiomyocyte apoptosis and the protective effects of carvedilol. Methods Find Exp Clin Pharmacol 2002;24:403-412.

2 Brinkmann V, Reichard U, Goosmann C, Fauler B, Uhlemann Y, Weiss DS, Weinrauch Y, Zychlinsky A: Neutrophil extracellular traps kill bacteria. Science 2004;303:15321535 .

3 Brinkmann V, Zychlinsky A: Neutrophil extracellular traps: is immunity the second function of chromatin? J Cell Biol 2012;198: 773-783.

4 Papayannopoulos V, Zychlinsky A: NETs: a new strategy for using old weapons. Trends Immunol 2009;30:513-521.

5 Demers M, Krause DS, Schatzberg D, Martinod K, Voorhees JR, Fuchs TA, Scadden DT, Wagnera DD: Cancers predispose neutrophils to release extracellular DNA traps that contribute to cancer-associated thrombosis. Proc Natl Acad Sci USA 2012;109:1307613081 .

6 Lin AM, Rubin CJ, Khandpur R, Wang JY, Riblett M, Yalavarthi S, Villanueva EC, Shah P, Kaplan MJ, Bruce AT: Mast cells and neutrophils release IL-17 through extracellular trap formation in psoriasis. J Immunol 2011; 187:490-500.
7 de Boer OJ, Li X, Teeling P, Mackaay C, Ploegmakers HJ, van der Loos CM, Daemen MJ, de Winter RJ, van der Wal AC: Neutrophils, neutrophil extracellular traps and interleukin-17 associate with the organisation of thrombi in acute myocardial infarction. Thromb Haemost 2013;109:290-297.

8 Barrett JA, Derian CK, Swillo RS, Woltmann $\mathrm{RF}$, Perrone MH: The role of the neutrophil and formed elements of the blood in an in vitro model of reperfusion injury. Mediators Inflamm 1993;2:85-92.

9 Ullrich O, Reinheckel T, Sitte N, Hass R, Grune T, Davies KJ: Poly-ADP ribose polymerase activates nuclear proteasome to degrade oxidatively damaged histones. Proc Natl Acad Sci USA 1999;96:6223-6228.

10 Knepper-Nicolai B, Savill J, Brown S: Constitutive apoptosis in human neutrophils requires synergy between calpains and the proteasome downstream of caspases. J Biol Chem 1998;273:30530-30536.

11 Campbell B, Adams J, Shin YK, Lefer AM: Cardioprotective effects of a novel proteasome inhibitor following ischemia and reperfusion in the isolated perfused rat heart. J Mol Cell Cardiol 1999;31:467-476.
12 Chitra S, Nalini G, Rajasekhar G: The ubiquitin proteasome system and efficacy of proteasome inhibitors in diseases. Int J Rheum Dis 2012;15:249-260.

13 Genin E, Reboud-Ravaux M, Vidal J: Proteasome inhibitors: recent advances and new perspectives in medicinal chemistry. Curr Top Med Chem 2010;10:232-256.

14 Lapponi MJ, Carestia A, Landoni VI, Rivadeneyra L, Etulain J, Negrotto S, Pozner RG, Schattner M: Regulation of neutrophil extracellular trap formation by anti-inflammatory drugs. J Pharmacol Exp Ther 2013;345:430437.

15 Dosenko VE, Nagibin VS, Tumanovskaya LV, Zagoriy VY, Moibenko AA, Vaage J: Proteasomal proteolysis in anoxia-reoxygenation, preconditioning and postconditioning of isolated cardiomyocytes. Pathophysiology 2006;13:119-125.

16 McCormack TA, Cruikshank AA, Grenier L, Melandri FD, Nunes SL, Plamondon L, Stein RL, Dick LR: Kinetic studies of the branched chain amino acid preferring peptidase activity of the 20 S proteasome: development of a continuous assay and inhibition by tripeptide aldehydes and clasto-lactacystin beta-lactone. Biochemistry 1998;37:7792-7800.
Proteasome Inhibitor Blocks NETosis and Prevents Cardiomyocyte Death
Pathobiology 2015;82:290-298 DOI: $10.1159 / 000440982$ 
17 Reinecke H, Zhang M, Bartosek T, Charles E: Survival, integration, and differentiation of cardiomyocyte grafts. Circulation 1999;100: 193-202.

18 Yost CC, Cody MJ, Harris ES, Thornton NL, McInturff AM, Martinez ML, Chandler NB, Rodesch CK, Albertine KH, Petti CA, Weyrich AS, Zimmerman GA: Impaired neutrophil extracellular trap (NET) formation: a novel innate immune deficiency of human neonates. Blood 2009;113:6419-6427.

19 Zhu J, Zhang H, Guo T, Li W, Li H, Zhu Y, Huang S: Quantitative proteomics reveals differential biological processes in healthy neonatal cord neutrophils and adult neutrophils. Proteomics 2014;14:1688-1697.

20 Kobayashi SD, Voyich MJ, Somerville GA, Braughton KR, Malech HL, Musser JM, DeLeo FR: An apoptosis-differentiation program in human polymorphonuclear leukocytes facilitates resolution of inflammation. J Leukoc Biol 2003;73:315-322.

21 Nolan B, Kim R, Duffy A, Sheth K, De M, Miller C, Chari R, Bankey P: Inhibited neutrophil apoptosis: proteasome dependent NF- $\mathrm{kB}$ translocation is required for TRAF-1 synthesis. Shock 2000;14:290-294.
22 Lush CW, Kvietys PR: Microvascular dysfunction in sepsis. Microcirculation 2000;7: 83-101.

23 Neviere RR, Cepinskas G, Madorin WS Hoque N, Karmazyn M, Sibbald WJ, Kvietys PR: LPS pretreatment ameliorates peritonitisinduced myocardial inflammation and dysfunction: role of myocytes. Am J Physiol 1999; 277:H885-H892.

24 Madorin WS, Rui T, Sugimoto N, Handa O, Cepinskas G, Kvietys PR: Cardiac myocytes activated by septic plasma promote neutrophil transendothelial migration: role of platelet-activating factor and the chemokines LIX and KC. Circ Res 2004;94:944-951.

25 Buerke M, Prüfer D, Dahm M, Oelert H, Meyer J, Darius H: Blocking of classical complement pathway inhibits endothelial adhesion molecule expression and preserves ischemic myocardium from reperfusion injury. J Pharmacol Exp Ther 1998;86:429-438.

26 Carmona-Rivera C, Zhao W, Yalavarthi S, Kaplan MJ: Neutrophil extracellular traps induce endothelial dysfunction in systemic lupus erythematosus through the activation of matrix metalloproteinase-2. Ann Rheum Dis 2015;74:1417-1424.
27 Ge L, Zhou X, Ji WJ, Lu RY, Zhang Y, Zhang YD, Ma YQ, Zhao JH, Li YM: Neutrophil extracellular traps in ischemia-reperfusion injury-induced myocardial no-reflow: therapeutic potential of DNase-based reperfusion strategy. Am J Physiol Heart Circ Physiol 2015;308:H500-H509.

28 Savchenko AS, Martinod K, Seidman MA, Wong SL, Borissoff JI, Piazza G, Libby P, Goldhaber SZ, Mitchell RN, Wagner DD: Neutrophil extracellular traps form predominantly during the organizing stage of human venous thromboembolism development. J Thromb Haemost 2014;12:860-870.

29 Oklu R, Albadawi H, Jones JE, Yoo HJ, Watkins MT: Reduced hind limb ischemia-reperfusion injury in Toll-like receptor-4 mutant mice is associated with decreased neutrophil extracellular traps. J Vasc Surg 2013;58:16271636.

30 Yu X, Kem DC: Proteasome inhibition during myocardial infarction. Cardiovasc Res 2010; $85: 312-320$. 